\title{
Crystallographic Directions of Zero Thermal Expansion in Anisotropic Oxides
}

Scott J. McCormack, Will Wheeler, Waltraud M. Kriven

Department of Materials Science and Engineering, University of Illinois at Urbana-Champaign, Illinois 61801, USA.

Oxide materials tend to have anisotropic crystal structures, which results in material properties being dependant on direction. Typically, oxide materials have positive thermal expansion coefficients. However, it has been observed that some directions can have negative thermal expansion coefficients over certain temperature ranges. These oxide materials, which exhibit directions of positive and negative thermal expansion, will also have a particular direction in which the thermal expansion is zero. By using the Quadrupole Lamp Furnace (QLF) developed in the Kriven group, high temperature in-situ x-ray diffraction has been performed at the National Synchrotron Light Source II (NSLSII) X-ray powder diffraction beamline (XPD - 28-ID) to track these directions of zero thermal expansion in orthorhombic $\mathrm{HfTiO}_{4}$ and $\mathrm{ZrTiO}_{4}$. $\mathrm{These}$ measurements are important for identifying materials which will be dimensionally accurate at elevated temperatures. 